Check for updates

Cite this: Chem. Sci., 2019, 10, 898

๑ All publication charges for this article have been paid for by the Royal Society of Chemistry

Received 16th September 2018 Accepted 30th October 2018

DOI: $10.1039 / c 8 s c 04120 b$

rsc.li/chemical-science

\section{X-ray Raman optical activity of chiral molecules $\uparrow$}

\author{
Jérémy R. Rouxel, (D) $t^{\star a}$ Yu Zhang (D) $t^{\star b}$ and Shaul Mukamel ${ }^{\star c d}$
}

Resonant and off-resonant Raman Optical Activity signals in the X-ray regime (XROA) are predicted. $\mathrm{XROA}$ is a chiral-sensitive variant of the spontaneous Resonant Inelastic Scattering (RIXS) signal. Thanks to the highly localized nature of core excitations, these signals provide a direct probe of local chirality with high sensitivity to the molecular structure. We derive sum-over-states expressions for frequency domain XROA signals and apply them to tyrosine at the nitrogen and oxygen $\mathrm{K}$-edges. Time-resolved extensions of ROA made possible by using additional pulses are briefly outlined.

\section{Introduction}

The recent development of intense X-ray light from synchrotron or X-ray free electron lasers (XFELs) ${ }^{1,2}$ makes it possible to extend well established infrared and optical nonlinear spectroscopic techniques ${ }^{3-6}$ to the X-ray regime. One notable advantage is that the light-matter interaction of $\mathrm{X}$-ray light resonant with core excitations in molecules is highly spatially localized and element specific. Signals specifically targeting molecular chirality thus offer important structural information. ${ }^{7}$ Chirality is crucial for the biological activity of biomolecules and has been extensively studied using visible and IR techniques such as circular dichroism (CD), optical rotation ${ }^{8}$ and Raman Optical Activity (ROA)., ${ }^{9,10}$ Chiral signals are intrinsically weak and considerable effort has been made to increase their magnitude ${ }^{11,12}$ which is vital, e.g. for sensor applications. ${ }^{13,14}$

ROA employs circularly polarized light to measure the vibrational or electronic optical activity and is routinely used in the infrared ${ }^{15}$ and the visible ${ }^{16}$ regimes to obtain information on the absolute configuration of small chiral molecules and on the secondary and tertiary structure of proteins. This is particularly important for drug design since many important drugs are chiral. The X-ray extension of ROA should also provide favorable scaling since it increases as $\omega^{5}$ compared to the conventional Raman background that scales as $\omega^{4} \cdot{ }^{17}$ Expressions for XROA

${ }^{a}$ Ecole Polytechnique Fédérale de Lausanne, Laboratoire de Spectroscopie Ultrarapide, Faculté des Sciences de Base, ISIC-BSP, CH-1015 Lausanne, Switzerland. E-mail: jrouxel@uci.edu

${ }^{\circ}$ PULSE Institute, SLAC National Accelerator Laboratory, Menlo Park, CA 94025, USA. E-mail: yzhang15@slac.stanford.edu

${ }^{c}$ Department of Chemistry, University of California, Irvine, CA 92697, USA. E-mail: smukamel@uci.edu

${ }^{d}$ Department of Physics and Astronomy, University of California, Irvine, CA 92697, USA $\dagger$ Electronic supplementary information (ESI) available. See DOI: $10.1039 / \mathrm{c} 8 \mathrm{sc} 04120 \mathrm{~b}$

† J. R. Rouxel and Y. Zhang have equal contributions. signals are derived in Section 2 and simulation results of the amino acid tyrosine are presented and analyzed in Section 3. Our findings are summarized in Section 4.

\section{The X-ray Raman optical activity signal}

ROA has its origin in the pioneering work of Barron ${ }^{16,18,19}$ who demonstrated that the spontaneous Raman scattering intensity induced by circularly polarized light from chiral enantiomers is different. The ROA signal is usually measured by taking the difference between spontaneous Raman spectra induced by left and right polarized light at different scattering angles. The detector then measures all polarizations of the scattered light. This configuration is shown in Fig. 1. ROA signals can also be acquired in other ways by detecting, for example, the difference between the left and right polarized components of the scattered light when the incident light is linearly polarized or unpolarized. ${ }^{16}$ Mixed schemes in which both the polarization of the incoming light and the scattered light are varied have been used as well. By varying the polarization and detecting the signal at

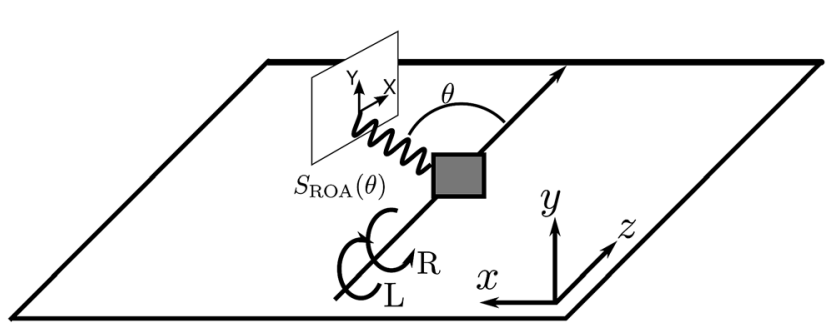

Fig. 1 The ROA measurement scheme. Left and right circularly polarized $X$-ray pulses propagating along the $z$ direction generate two Raman signals which are recorded as a function of the scattering angle. Their small difference constitutes the ROA signal, eqn (9). The detector polarization basis by $X$ and $Y$ provides an extra degree of freedom for the measurement. 
different scattering angles, it is possible to isolate different tensor components of the rotationally averaged chiral signal. ${ }^{16}$

ROA with visible light is by now a well established experimental technique for probing the optical activity of the vibrational manifold. ${ }^{9}$ The electronic chirality is most commonly observed by visible or UV CD. ${ }^{20,21}$ XROA measures the optical activity of the valence electronic excited manifold accessible through intermediate core electronic excitations. For the setup shown in Fig. 1, the relevant matter correlation function is a pseudo-tensor involving a magnetic dipole or an electric quadrupole interaction. The sum of these scattering intensities depends only on true tensor quantities (electric dipole-electric dipole interactions). The ROA signals can be directly deduced from the loop diagrams shown in Fig. 2 and are usually normalized by the sum of the spectra (eqn (9)).

In this paper, we study the difference in the Raman signal induced by the incoming left and right polarizations. The radiation/matter coupling is given by the multipolar Hamiltonian $^{22}$ truncated at the electric quadrupole

$$
H_{\mathrm{int}}(t)=-\boldsymbol{\mu} \cdot \mathbf{E}_{\mathrm{s}}-\boldsymbol{m} \cdot \mathbf{B}_{\mathrm{s}}-\boldsymbol{q} \cdot \nabla \mathbf{E}_{\mathrm{s}}
$$

Fig. 2(a), (b) and (c) represent the non-chiral electric dipole contribution, the magnetic dipole and the electric quadrupole, respectively. We shall denote the detected spontaneous Raman intensity by $S_{\mathrm{RAM}}\left(\omega_{\mathrm{X}}, \omega_{\mathrm{s}}, \theta, \mathbf{e}_{\mathrm{L} / \mathrm{R}}, \mathbf{e}_{\mathrm{s}}\right)$, where $\omega_{\mathrm{X}}$ is the incident pulse frequency, $\omega_{\mathrm{s}}$ is the detected frequency, $\theta$ is the angle between the incident pulse and the detected light, and $\mathbf{e}_{\mathrm{L} / \mathrm{R}}$ and $\mathbf{e}_{\mathrm{s}}$ are the incident and diffracted pulse polarizations.

The XROA signal is given by the difference between left and right incoming polarizations:

$$
\begin{aligned}
S_{\mathrm{XROA}}\left(\omega_{\mathrm{X}}, \omega_{\mathrm{s}}, \theta, \mathbf{e}_{\mathrm{s}}\right)= & S_{\mathrm{RAM}}\left(\omega_{\mathrm{X}}, \omega_{\mathrm{s}}, \theta, \mathbf{e}_{\mathrm{L}}, \mathbf{e}_{\mathrm{s}}\right) \\
& -S_{\mathrm{RAM}}\left(\omega_{\mathrm{X}}, \omega_{\mathrm{s}}, \theta, \mathbf{e}_{\mathrm{R}}, \mathbf{e}_{\mathrm{s}}\right)
\end{aligned}
$$

Without loss of generality, in an isotropic sample, we assume that the incident pulse propagates along the $z$ axis (this implies that $\mathbf{k}_{\mathrm{X}}=\mathbf{e}_{z}$ ) and that the scattered Raman signal is measured in the $x z$ plane (see Fig. 1).
The spontaneous Raman signal is defined as the change of photon number emitted in the detected direction

$$
\begin{aligned}
S_{\mathrm{RAM}}\left(\omega_{\mathrm{s}}\right)= & \int \mathrm{d} t \frac{\mathrm{d}}{\mathrm{d} t}\left\langle\mathbf{E}_{\mathrm{s}}^{\dagger}(\mathbf{r}, t) \cdot \mathbf{E}_{\mathrm{s}}(\mathbf{r}, t)\right\rangle+\left\langle\mathbf{E}_{\mathrm{s}}^{\dagger}(\mathbf{r}, t) \cdot \mathbf{B}_{\mathrm{s}}(\mathbf{r}, t)\right\rangle \\
& +\left\langle\mathbf{B}_{\mathrm{s}}^{\dagger}(\mathbf{r}, t) \cdot \mathbf{E}_{\mathrm{s}}(\mathbf{r}, t)\right\rangle
\end{aligned}
$$

We shall neglect the magnetic field contribution because it is orders of magnitude smaller. ${ }^{23}$ The time derivative can be calculated by using the expectation value of the Heisenberg equation of motion of the number operator.

$$
\begin{aligned}
\frac{\mathrm{d}}{\mathrm{d} t}\left\langle\mathbf{E}_{\mathrm{s}}^{\dagger} \cdot \mathbf{E}_{\mathrm{s}}\right\rangle= & \frac{i}{\hbar}\left\langle\left[-\mu \cdot\left(\mathbf{E}_{\mathrm{s}}^{\dagger}+\mathbf{E}_{\mathrm{s}}\right)-m \cdot\left(\mathbf{B}_{\mathrm{s}}^{\dagger}+\mathbf{B}_{\mathrm{s}}\right)\right.\right. \\
& \left.\left.-q \cdot\left(\nabla \mathbf{E}_{\mathrm{s}}^{\dagger}+\nabla \mathbf{E}_{\mathrm{s}}\right), \mathbf{E}_{\mathrm{s}}^{\dagger} \cdot \mathbf{E}_{\mathrm{s}}\right]\right\rangle
\end{aligned}
$$

The commutator can be calculated in a straightforward manner using the definition of the $\mathbf{E}_{\mathrm{s}}$ and $\mathbf{B}_{\mathrm{s}}$ operators

$$
\begin{gathered}
\mathbf{E}_{\mathrm{s}}=i \sqrt{\frac{\hbar \omega_{\mathrm{s}}}{2 \varepsilon_{0} \Omega}} a_{\mathrm{s}} \mathbf{e}_{\mathrm{s}} \mathrm{e}^{\mathrm{i}\left(\mathbf{k}_{\mathrm{s}} \cdot \mathbf{r}-\omega_{\mathrm{s}} t\right)} \\
\mathbf{B}_{\mathrm{s}}=\frac{i}{c} \sqrt{\frac{\hbar \omega_{\mathrm{s}}}{2 \varepsilon_{0} \Omega}} a_{\mathrm{s}}\left(\hat{\mathbf{k}}_{\mathrm{s}} \times \mathbf{e}_{\mathrm{s}}\right) \mathrm{e}^{\mathrm{i}\left(\mathbf{k}_{\mathrm{s}} \cdot \mathbf{r}-\omega_{\mathrm{s}} t\right)}
\end{gathered}
$$

where $\mathbf{k}_{\mathrm{s}}$ is the wavevector of the emitted photon, $\boldsymbol{\Omega}$ is the quantization volume and we have the boson commutation

$$
\left[a_{\mathrm{r}}, a_{\mathrm{s}}^{\dagger}\right]=\delta_{\mathrm{rs}}
$$

Upon evaluating the commutator, the Raman signal (eqn (3)) becomes

$$
\begin{aligned}
S_{\mathrm{RAM}}\left(\omega_{\mathrm{s}}\right)= & \frac{2}{\hbar} \frac{\hbar \omega_{\mathrm{s}}}{2 \varepsilon_{0}} \mathfrak{\Im} \int \mathrm{d} t\left\langle\mathbf{E}_{\mathrm{s}}^{\dagger}(t) \cdot \boldsymbol{\mu}(t)+\mathbf{B}_{\mathrm{s}}^{\dagger}(t) \cdot \boldsymbol{m}(t)\right. \\
& \left.+\nabla \mathbf{E}_{\mathrm{s}}^{\dagger}(t) \cdot \boldsymbol{q}(t) \mathrm{e}^{-i / \hbar \int \mathrm{d} \tau H_{\text {int }}(\tau)}\right\rangle
\end{aligned}
$$

where $\Re$ and $\mathfrak{J}$ denote the real and imaginary parts respectively. To obtain the ROA signal, we expand the exponent to second order in the incident field. Three terms then contribute to the signals. The leading term is given by the interaction with the electric dipoles only (diagram (a)) and does not contribute to the

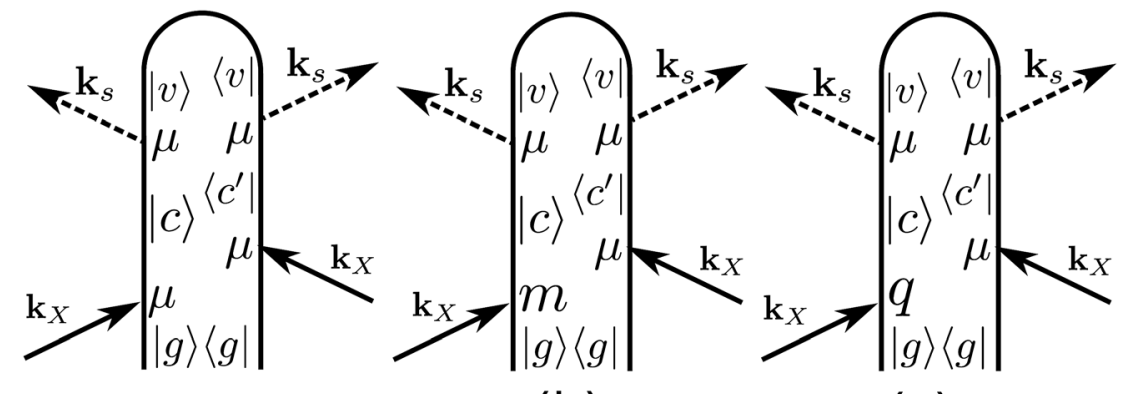

(a)

(b)

(c)

Fig. 2 Loop diagrams for the electric dipole (a), magnetic dipole (b) and electric quadrupole (c) contributions to the ROA signal. We only plot one of the four diagrams involving the magnetic dipole contribution to the signal. The other three can be obtained for (b) by permuting the magnetic dipole interaction with the electric dipole ones. Similarly, there are four diagrams for the electric quadrupole interaction (c). 
ROA signal since it vanishes upon rotational averaging. Diagram (b) contains one magnetic dipole and diagram (c) has one electric quadrupole interaction. pulse tuned at the $\mathrm{N}\left(\omega_{\mathrm{X}} \sim 389-397 \mathrm{eV}\right)$ and $\mathrm{O}\left(\omega_{\mathrm{X}} \sim 519-526 \mathrm{eV}\right)$ K-edges. The incoming $\mathrm{X}$-rays are thus resonant with the excited transitions. The signals on D-tyrosine are identical but with an

$$
\begin{aligned}
S_{\mathrm{RAM}} & \left(\omega_{\mathrm{X}}, \omega_{\mathrm{s}}, \theta, \mathbf{e}_{\mathrm{L} / \mathrm{R}}, \mathbf{e}_{\mathrm{s}}\right)=\left(\frac{\hbar \omega_{\mathrm{s}}}{2 \epsilon_{0} \Omega}\right)^{2} \Re \int \mathrm{d} t \mathrm{~d} t_{3} \mathrm{~d} t_{2} \mathrm{~d} t_{1} \mathrm{~d} \mathbf{r}_{4} \mathrm{~d} \mathbf{r}_{3} \mathrm{~d} \mathbf{r}_{2} \mathrm{~d} \mathbf{r}_{1} \times \mathrm{e}^{i \mathbf{k}_{\mathrm{X}} \cdot\left(\mathbf{r}_{1}-\mathbf{r}_{3}\right)} \mathrm{e}^{-i \mathbf{k}_{\mathrm{s}} \cdot\left(\mathbf{r}_{2}-\mathbf{r}_{4}\right)} \mathrm{e}^{i \omega_{\mathrm{X}}\left(t-t_{3}\right)} \mathrm{e}^{-i \omega_{\mathrm{X}}\left(t-t_{3}-t_{2}-t_{1}\right)} \mathrm{e}^{-i \omega_{\mathrm{s}} t} \mathrm{e}^{i \omega_{\mathrm{s}}\left(t-t_{3}-t_{2}\right)} \\
& \times\left[\mathbf{E}_{\mathrm{X}}^{*}\left(\mathbf{r}_{3}, t-t_{3}\right) \otimes \mathbf{e}_{\mathrm{s}}^{*} \otimes \mathbf{e}_{\mathrm{s}} \otimes \mathbf{E}_{\mathrm{X}}\left(\mathbf{r}_{1}, t-t_{3}-t_{2}-t_{1}\right)\left\langle\boldsymbol{\mu}\left(t_{3}\right) \boldsymbol{\mu}\left(t_{2}\right) \boldsymbol{\mu}\left(t_{1}\right) \boldsymbol{\mu}(0)\right\rangle\right. \\
& +\mathbf{E}_{X}^{*}\left(\mathbf{r}_{3}, t-t_{3}\right) \otimes \mathbf{e}_{\mathrm{s}}^{*} \otimes \mathbf{e}_{\mathrm{s}} \otimes \mathbf{B}_{\mathrm{X}}\left(\mathbf{r}_{1}, t-t_{3}-t_{2}-t_{1}\right)\left\langle\boldsymbol{\mu}\left(t_{3}\right) \boldsymbol{\mu}\left(t_{2}\right) \boldsymbol{\mu}\left(t_{1}\right) \boldsymbol{m}(0)\right\rangle+\mathbf{E}_{\mathrm{X}}^{*}\left(\mathbf{r}_{3}, t-t_{3}\right) \otimes \mathbf{e}_{\mathrm{s}}^{*} \otimes \mathbf{e}_{\mathrm{s}} \nabla \mathbf{E}_{\mathrm{X}}\left(\mathbf{r}_{1}, t-t_{3}-t_{2}-t_{1}\right) \\
& \left.\times\left\langle\boldsymbol{\mu}\left(t_{3}\right) \boldsymbol{\mu}\left(t_{2}\right) \boldsymbol{\mu}\left(t_{1}\right) \boldsymbol{q}(0)\right\rangle+\text { permutations of magnetic and quadrupole interactions }\right] .
\end{aligned}
$$

where the $\mathbf{e}_{\mathrm{L} / \mathrm{R}}$ dependence of the signal is contained in the vector components of $\mathrm{E}_{\mathrm{X}}$ and $\mathrm{B}_{\mathrm{X}}$. The three terms in brackets correspond to diagrams (a), (b) and (c), respectively in Fig. 2 and all operators evolve with the free molecular Hamiltonian in the interaction picture. Eqn (9) allows us to calculate timeresolved signals induced by broadband pulses. In that case, the spatial and temporal integrals can be then expressed as a Fourier transform. In this paper, we focus on ROA signals in the frequency domain measured with monochromatic light excitation. The total XROA signal is given by the sum of the magnetic and the quadrupole contributions:

$$
S_{\mathrm{XROA}}\left(\omega_{\mathrm{X}}, \omega_{\mathrm{s}} ; \theta, \mathbf{e}_{\mathrm{s}}\right)=S_{\mathrm{XROA}}^{\mathrm{mag}}\left(\omega_{\mathrm{X}}, \omega_{\mathrm{s}} ; \theta, \mathbf{e}_{\mathrm{s}}\right)+S_{\mathrm{XROA}}^{\mathrm{quad}}\left(\omega_{\mathrm{X}}, \omega_{\mathrm{s}} ; \theta, \mathbf{e}_{\mathrm{s}}\right)
$$

Sum-over-states expressions for eqn (9) are given in Appendix B.

\section{XROA signals of tyrosine}

We have calculated the XROA signal, eqn (9), for a randomly oriented ensemble of L-tyrosine (Fig. 3(a)) with an X-ray incident opposite sign and thus are not displayed. We focus on the zwitterionic structure (see Fig. 3(a)) of the molecule in water since this is its dominant form at physiological $\mathrm{pH}$. The geometry was optimized at the $\mathrm{B} 3 \mathrm{LYP}^{24,25} /$ aug-cc-pVTZ ${ }^{26,27}$ level of theory using the Gaussian program package. ${ }^{28}$ The water solvent effect was considered with the PCM model. ${ }^{29-31}$ All quantities needed for XROA signal calculations were calculated using the CD spectrum ${ }^{32-34}$ and the restricted excitation window time-dependent density functional (REWTDDFT) ${ }^{35-40}$ implementations in the quantum chemistry package NWChem ${ }^{\mathbf{4 1}}$ under the Tamm-Dancoff approximation (TDA). ${ }^{\mathbf{4 2} 44}$ The computational protocol for the relevant transition moments (electric and magnetic dipoles, electric quadrupoles) has been presented in ref. 40. The B3LYP hybrid functional and the cc-pVTZ basis set $^{26}$ were used for all NWChem calculations. The water solvent effect was included using the COSMO model. ${ }^{45}$ It is a common practice to apply constant shifts to the calculated core excitation energies from TDDFT to match the experimental results. Since in this study only the relative positions of spectroscopic features matter, we retain all original core excitation energies from the TDDFT calculations. We adopted a tight SCF convergence criterion (a)

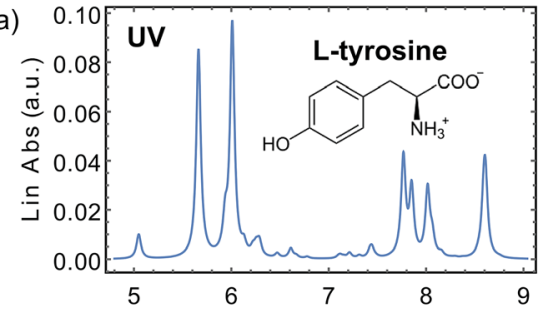

(b)

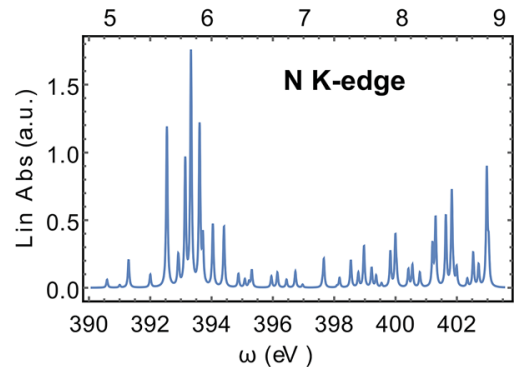

(c)

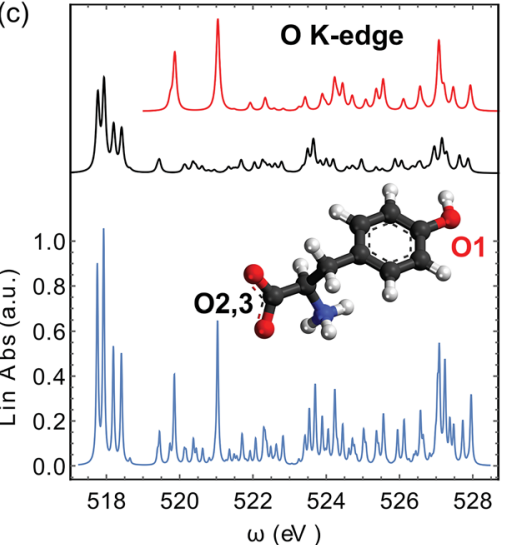

Fig. 3 Calculated spectra of L-tyrosine in its zwitterionic form. (a) UV-vis spectrum. (b) N K-edge X-ray absorption spectrum. (c) O K-edge X-ray absorption spectrum. The 3D molecular model is also shown and the $x, y, z$ axes are marked in red, green and blue, respectively. The oxygen atom in the phenol group is labelled $\mathrm{O}_{1}$ and the other two in the carbonyl group are labelled $\mathrm{O}_{2,3}$. The separate contributions of these two types of $\mathrm{O}$ atoms to the total signal (in light blue) are plotted in red and black, respectively. The signal contributions are normalized to different scales. 
(energy change of SCF cycles $<10^{-8}$ a.u.) and an ultrafine grid in all NWChem calculations.

It is well known that TDDFT-TDA in general produces excitation energies of identical or even better quality of full TDDFT, but possibly worse oscillator strengths because of violation of the sum rule. ${ }^{46,47}$ However, previous TDDFT-TDA studies on core spectroscopy, which is the major topic of this manuscript, showed that TDDFT-TDA and full TDDFT gave almost indistinguishable results. ${ }^{39,48}$ TDDFT-TDA is computationally much cheaper than full TDDFT, which facilitates its use in large systems. Moreover, it handles triplet instabilities much better than full TDDFT. ${ }^{49}$ In addition, it also gives better nonadiabatic couplings by reducing the local-density-approximation (LDA) error ${ }^{50}$ With all the considerations above, we use TDDFT-TDA in this study.

Under the multipolar expansion, only the first non-vanishing multipole is origin invariant. Thus, in the present work, the electric quadrupoles and the magnetic dipoles are origin dependent. ${ }^{51}$ Indeed, the spectroscopic observables calculated from exact wave functions must be independent of the origin. ${ }^{52}$ However, numerical truncation of the basis set can induce origin-dependence of the spectroscopic observables. A possible solution is to use Gauge Invariant Atomic Orbitals (GIAOs) or London orbitals. ${ }^{34,53-55}$ Alternatively, as shown by Reiher et al., ${ }^{\mathbf{2 2}}$ by evaluating multipoles in the velocity gauge, the corresponding spectroscopic tensors are origin-independent even with a truncated basis. The behavior of the spectroscopic
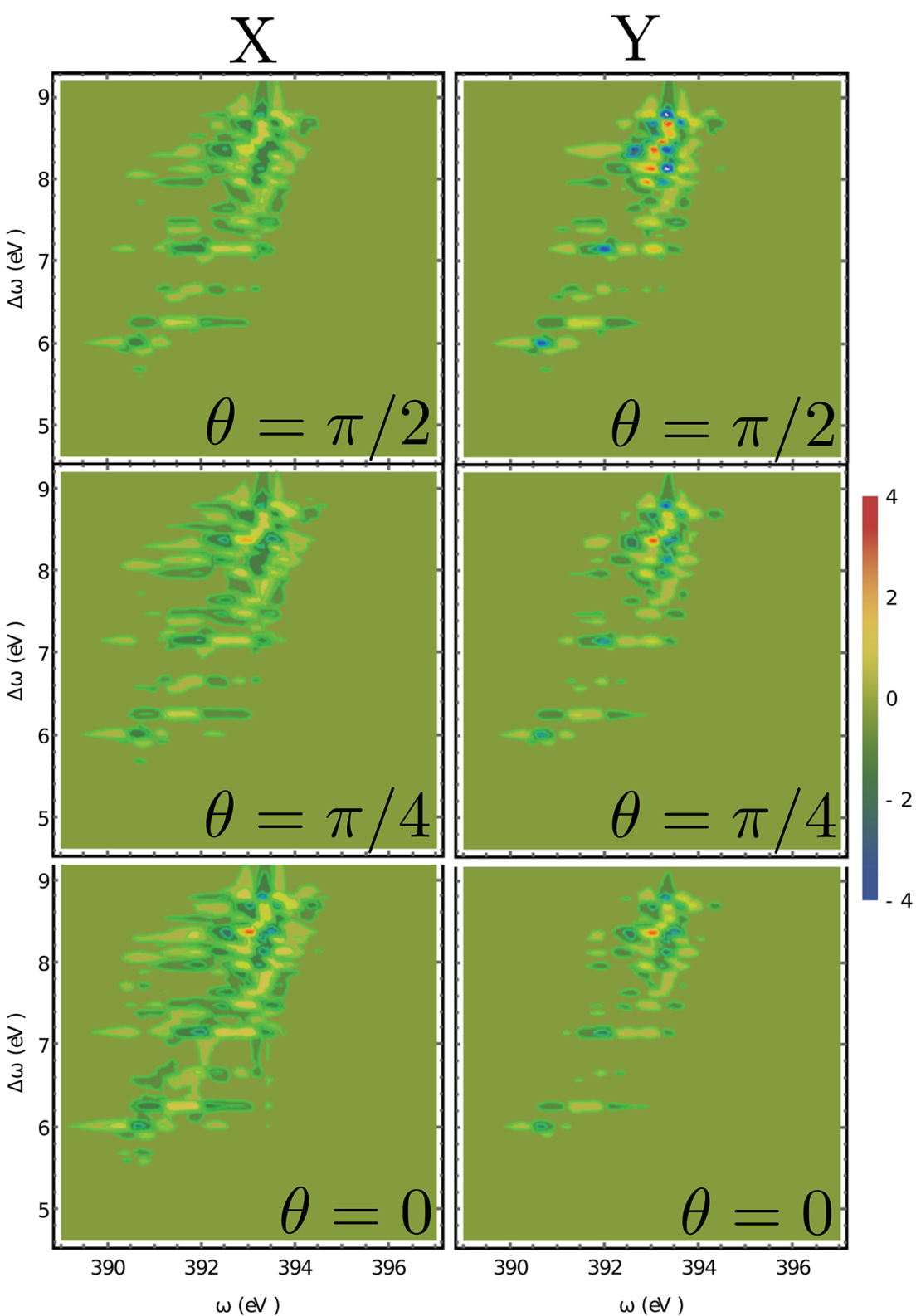

Fig. 4 2D XROA signals (eqn (9)) displayed vs. the incoming frequency $\omega_{X}$ and the Raman frequency shift $\Delta \omega=\omega_{X}-\omega_{\mathrm{s}}$ at the $\mathrm{N}$ K-edge for various scattering angles. The left and right columns represent $X$ and $Y$ polarizations of the detected photons in the detector frame. 
tensors upon changing the coordinates is given in Appendix $\mathrm{C}$ for completeness. To obtain origin-independent XROA signals, in this study we employed electric transition dipoles in the velocity gauge and kept the electric transition quadrupoles in the length gauge as their relevant spectroscopic tensors are origin-independent in both gauges. ${ }^{52}$

The calculated absorption spectra from TDDFT are displayed in the near UV range (Fig. 3(a)), at the N K-edge (Fig. 3(b)) and the $\mathrm{O}$ K-edge (Fig. 3(c)). Tyrosine has three $\mathrm{O}$ atoms, one in the phenol group and the other two in the carbonyl group. The carbonyl $\mathrm{O}$ atoms are chemically indistinguishable. Thus we separate the three $\mathrm{O}$ atoms into two groups, in which the phenol $\mathrm{O}$ atom is labelled $\mathrm{O}_{1}$ and the carbonyl $\mathrm{O}$ atoms are labelled $\mathrm{O}_{2,3}$ (Fig. 3). In our analysis, we have also decomposed the O K-edge spectrum into the contributions from $\mathrm{O}_{1}$ and $\mathrm{O}_{2,3}$, and plotted them in red and black in Fig. 3(c).

The XROA signal at the N K-edge $v s$. the incoming plane wave frequency and of the Raman shift $\omega_{X}-\omega_{\mathrm{S}}$ is displayed in Fig. 4 for various scattering angles. The corresponding 2D Raman signal at the scattering angle of $\pi / 2$ is given in the ESI. $\dagger$ The ratio of the chiral contribution to the nonchiral one is $1.7 \%$ at the main peak of the spectrum $(392.2 \mathrm{eV}$ excitation and $8.4 \mathrm{eV}$ Raman shift). The former provides spectroscopic information on the core-excited manifold while the latter covers the valence excited state manifold. The signals are given for both polarizations of detection $\mathrm{e}^{x}$ and $\mathrm{e}^{y}$ (see Fig. 1). Each panel depicts the signal acquired at various scattering angles between the incoming and emitted light. At $\theta=0$ (forward scattering), the signal is independent of the polarization. Due to the tight localization of the X-ray excitations in the vicinity of the core excited atom, strong peaks appear when the wave function overlap between the core-excited state and the valence excited state is high. Fig. 5 shows several slices of the N K-edge XROA at $\pi / 2$ scattering. The spectrum varies with $\theta$ while retaining the same structural information.

Fig. 6 depicts the total XROA signals calculated at various K-edges, nitrogen and two groups of oxygen atoms for both $\mathrm{X}$ and Y-polarization detection at a scattering angle of $\pi / 2$. The Xand Y-polarization detected signals show similar patterns. As can be seen in Section B-eqn (16), the ROA signal depends on multiple transitions and their orientations, hence there is no simple relationship between a single transition momentum and the signal. However, as a general rule, strong ROA signals require that the relevant core and valence excitations involve the same particle orbitals (since the corresponding transition moment is a single-electron operator), and the involved particle or hole orbital should be close to the chiral centers if they exist. We can take the strong $\mathrm{N}$ 1s ROA features as an example.
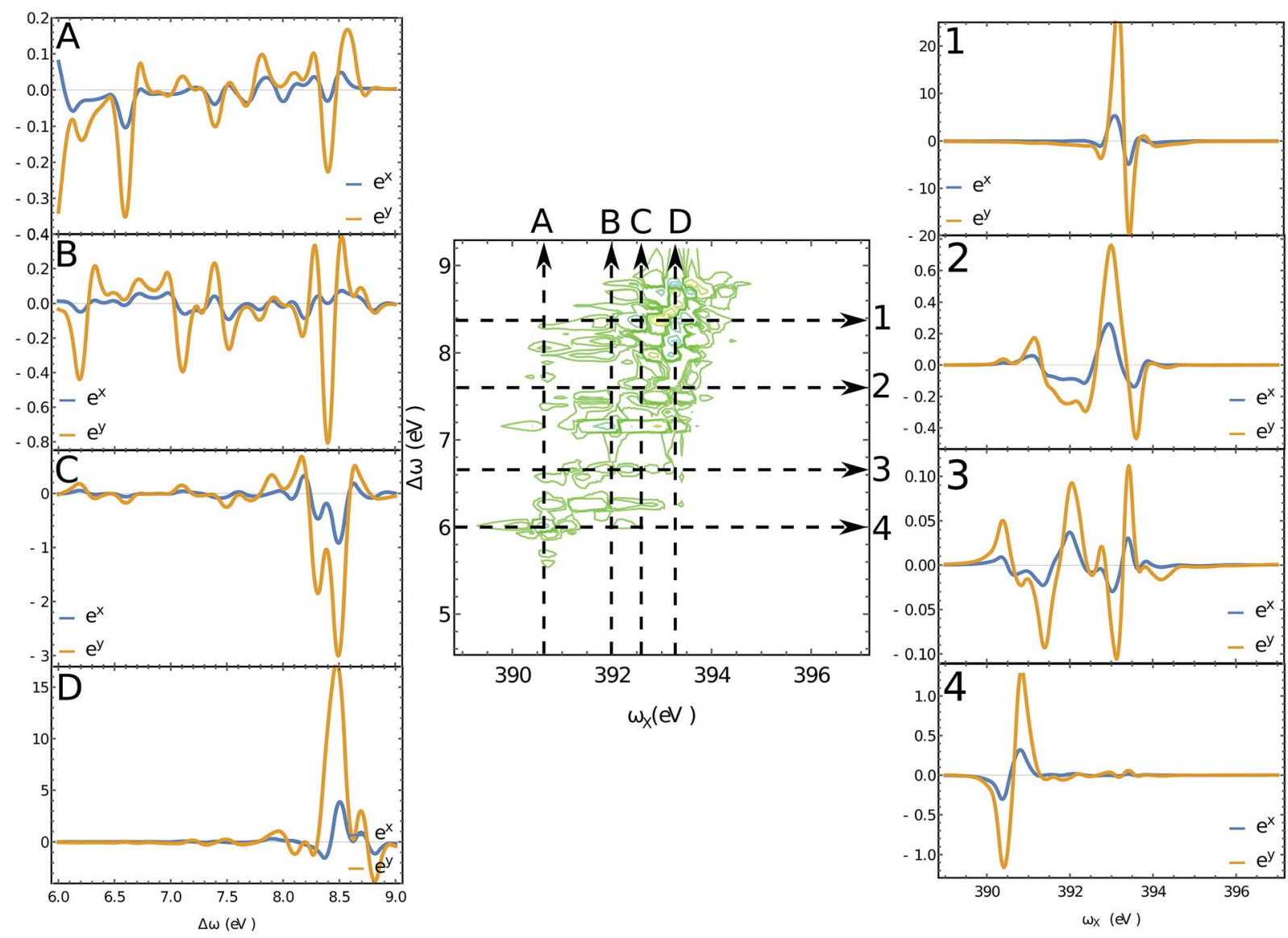

Fig. 5 Slices of the 2D XROA signals at the N K-edge, Fig. 4 for the scattering angle $\theta=\pi / 2$. The polarizations of the detected photons are shown in blue $\left(\mathrm{e}^{\mathrm{X}}\right)$ and orange $\left(\mathrm{e}^{\mathrm{Y}}\right)$. 

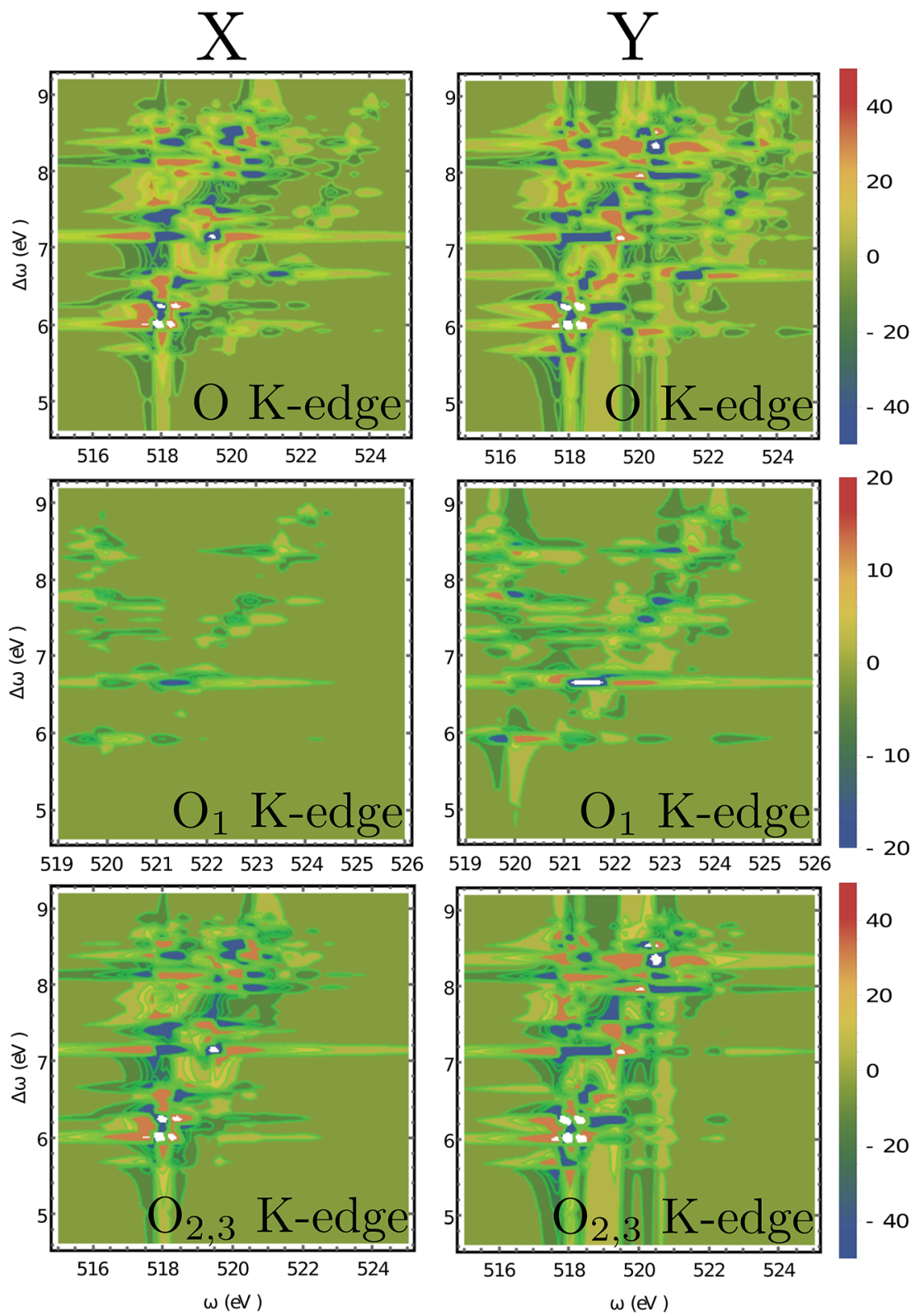

Fig. 6 2D XROA signals (eqn (9)) displayed vs. the incoming frequency $\omega_{X}$ and the frequency shift $\Delta \omega=\omega_{X}-\omega_{s}$ at various edges. $O_{1}$ refers to the oxygen atom in the $\mathrm{OH}$ group near the benzene ring while $\mathrm{O}_{2,3}$ are the two equivalent oxygen atoms at the carbonyl group. These two atoms are spectroscopically equivalent since the hydrogen atom can easily migrate between them. The scattering angle is $\pi / 2$.

Important molecular orbitals (MOs) (S1) and an analysis on relevant excitations (Table $\mathrm{S} 1 \dagger$ ) are provided in the ESI. $\dagger$ The strong feature around the point $(390.6,6.0) \mathrm{eV}$ involves the weak $\mathrm{N} 1 \mathrm{~s}$ core excitation at $390.6 \mathrm{eV}$ and the strong valence excitation at $6.0 \mathrm{eV}$. The two excitations couple through the LUMO, which is distributed on the benzene ring (see S1). Since the LUMO (L) is not close to the $\mathrm{N}$ atom, the corresponding X-ray absorption is weak (Fig. 3). However, the relevant valence hole orbital HOMO-3 (H-3) lies on the $\mathrm{N}$ atom as well as the chiral center, and the benzene ring, so the corresponding valence excitation and the chiral ROA signal is strong. Another strong ROA feature around the point $(393.4,8.4)$ eV can be explained similarly. The core excitation around $393.4 \mathrm{eV}$ couples with the valence excitation around $8.4 \mathrm{eV}$ through the $\mathrm{L}+7$ particle orbital. This orbital is delocalized over all atoms, and the corresponding valence hole orbital $\mathrm{H}-1$ carries the information regarding the chiral center.

The $\mathrm{O}$ 1s ROA signals are more complicated since there are two inequivalent $\mathrm{O}$ atoms in the molecule. The total $\mathrm{O}$ 1s ROA signals and the contributions from the $\mathrm{O}_{1}$ and $\mathrm{O}_{2,3}$ atoms are displayed in Fig. 6. As discussed in our previous work on X-ray $\mathrm{CD},{ }^{56}$ the chiral X-ray signal intensity depends on the distance between the X-ray chromophore and the molecular chiral center. This propensity is also clearly observed here by comparing the XROA signals of $\mathrm{O}_{1}$ and $\mathrm{O}_{2,3}$. One can see from 
Fig. 6 that the signals of $\mathrm{O}_{2,3}$ are much stronger than $\mathrm{O}_{1}$ signals since the carbonyl oxygens are closer to the chiral center. Taking the $x$-polarized detection as an example, $\mathrm{O}_{1}$ only shows a strong feature around the $(521.5,6.6) \mathrm{eV}$ point. The involved core excitation couples with the valence excitation through the orbital $\mathrm{L}+2$. We see that $\mathrm{L}+2$ almost covers the entire chiral center (S1) and is involved in many important core and valence transitions (Table S1†), thus it is the key orbital responsible for strong ROA signals. The strong $\mathrm{O}_{2,3}$ features around the points (518.2, 6.2) eV and $(519.4,7.2) \mathrm{eV}$ all involve $\mathrm{L}+2$. Alternatively, the $\mathrm{O}_{2,3}$ core excitations near $518 \mathrm{eV}$ also couple with the $6.0 \mathrm{eV}$ valence excitation through the $\mathrm{L}$ orbital. The other significant features can be analyzed in a similar manner.

Moreover, even though one can see the chemical shifts of $\mathrm{O}_{1}$ and $\mathrm{O}_{2,3}$ in the calculated linear X-ray absorption signals (Fig. 3(c), $\mathrm{O}_{1}$ has strong features near 520 and $521 \mathrm{eV}$, and $\mathrm{O}_{2,3}$ have strong features around $518 \mathrm{eV}$ ), the two spectra overlap around $520 \mathrm{eV}$ (note that the two spectra are plotted in different scales and the $\mathrm{O}_{2,3}$ signals are actually stronger than they appear in Fig. 3 (c) compared to the $\mathrm{O}_{1}$ signals). The large core hole lifetime broadening could produce a broad peak around $520 \mathrm{eV}$ which will complicate the assignment of the $\mathrm{O}$ peaks. However, the situation is different for the XROA signals. As shown in Fig. 6, the cross-peaks of $\mathrm{O}_{1}$ and $\mathrm{O}_{2,3}$ now have chemical shifts in both the core excitation and Raman shift energy dimensions. The various features of $\mathrm{O}_{1}$ and $\mathrm{O}_{2,3}$ can be better resolved in the valence excitation manifold, thanks to the much smaller lifetime broadening of the final valence excited

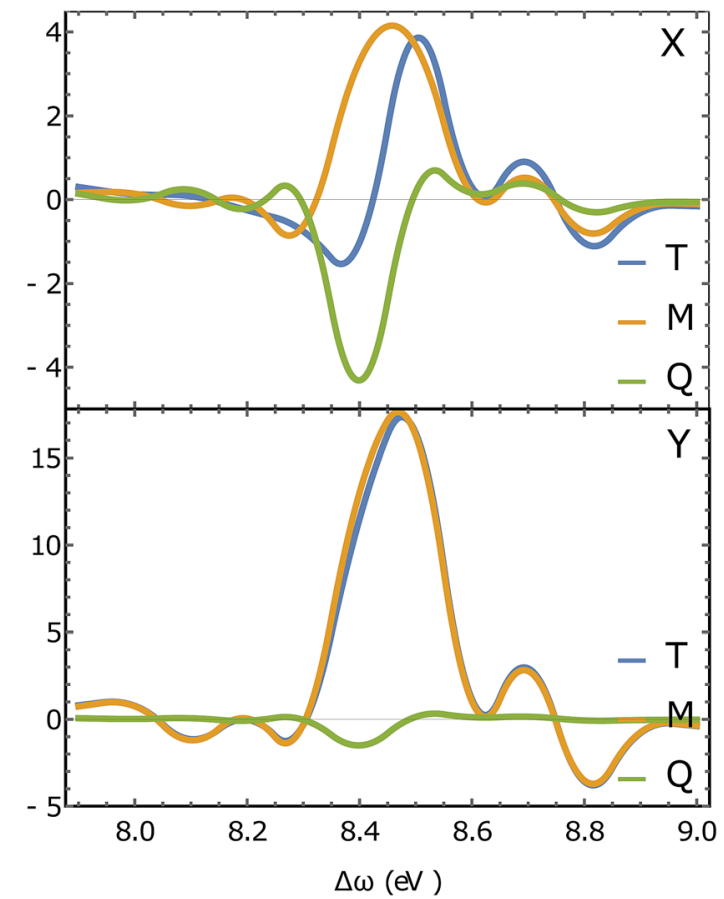

Fig. 7 Slices of the XROA signal at the N K-edge $\left(\omega_{\mathrm{x}}=393.25 \mathrm{eV}, \theta=\right.$ $\pi / 2$ for $x$-polarized detection (top) and $y$-polarized detection (bottom). The total XROA signal measured ( $T$, blue) is the sum of magnetic dipole ( $M$, orange) and electric quadrupole $(Q$, green) contributions. states. Therefore, XROA signals have advantages over XCD signals by better separating the contributions from different atoms to the chiral signal and resolving the local chirality information at a new level.

Fig. 7 depicts the magnetic and quadrupole contributions to the XROA signal at the $\mathrm{N}$ K-edge $\left(\omega_{\mathrm{X}}=393.25 \mathrm{eV}, \theta=\pi / 2\right)$, eqn (16), for $x$ and $y$ polarization of the signal. The total signal measured at the $\mathrm{N}$ K-edge is given by the sum of the magnetic and the electric contributions. For $x$-polarized detection, the total signal (blue curve) is a mixture of magnetic dipole and electric quadrupole contributions of similar weight. The $y$ polarized signal under the same conditions is dominated by the magnetic interaction. The polarization direction of detection along which the quadrupole contribution is important is determined by the geometric and electronic structure of the system, but the fact that this contribution varies significantly for different polarizations of detection is general. The sensitivity of XROA signals to the electric quadrupole interaction opens a new window to molecular chirality compared to XCD signals which do not depend on the electric quadrupole because of cancellation caused by rotational averaging.

\section{Conclusions}

The most common technique to study chirality with circularly polarized X-ray light is CD. ${ }^{56-58}$ We have recently shown how Xray $\mathrm{CD}$ may be used to locally probe chiral structures. In the present study we have simulated the XROA signals of L-tyrosine at the $\mathrm{N}$ and $\mathrm{O}$ K-edges. ROA is routinely used in the IR and visible regimes, but has not been reported yet for X-rays. The technique has been made feasible by the use of advanced X-ray sources.

One advantage of ROA spectroscopy is that the signals can be detected in a non-collinear geometry thus avoiding the strong background of an intense incoming X-ray beam. Moreover, like optical Raman signals, ROA signals may detect dark states in the corresponding absorption spectra because of different selection rules, and usually have better spectral resolution than their absorption counterparts. Unlike XCD, ROA also depends on the electric quadrupole interactions for randomly oriented molecules and can thus probe molecular chirality even when magnetic dipoles are weak, as has been demonstrated for tri$\mathrm{s}$ (ethylenediamine) cobalt(III) ion $\left(\mathrm{Co}(\mathrm{en}){ }_{3}{ }^{3+}\right) \cdot{ }^{59}$

The signal in eqn (9) was calculated for a monochromatic excitation. It will be interesting to extend this technique to the time-domain by using ultrashort X-ray pulses. Time-resolved extension can be achieved by adding an actinic pulse with a controlled delay in an optical pump/X-ray probe scheme. This can be done by starting with eqn (9) and expanding the exponent to higher orders in the incoming pulses in order to trigger some chiral dynamics. XROA can then be used as a novel detection mode on top of a nonlinear process that would be sensitive to local change of the chiral structure in the vicinity of an X-ray chromophore. Time-domain XROA should be a powerful spectroscopy tool to track chiral molecules in chemical reactions. 


\section{Appendix A rotationally averaged tensors}

A $n$-th rank tensor $T$ is rotationally averaged using the averaging tensor $I^{(n)}{ }^{60}$ The correlation tensor involving electric and magnetic dipoles are only fourth rank tensors for ROA and the ones involving electric quadrupole are of rank 5.

$$
\begin{aligned}
& I^{(4)}=\frac{1}{30}\left(\begin{array}{l}
\delta_{i_{1} i_{2}} \delta_{i_{1} i_{2}} \\
\delta_{i_{1} i_{3}} \delta_{i_{2} i_{4}} \\
\delta_{i_{1} i_{4}} \delta_{i_{2} i_{3}}
\end{array}\right)^{T}\left(\begin{array}{ccc}
4 & -1 & -1 \\
-1 & 4 & -1 \\
-1 & -1 & 4
\end{array}\right)\left(\begin{array}{l}
\delta_{\lambda_{1} \lambda_{2}} \delta_{\lambda_{1} \lambda_{2}} \\
\delta_{\lambda_{1} \lambda_{3}} \delta_{\lambda_{2} \lambda_{2}} \\
\delta_{\lambda_{1} \lambda_{4}} \delta_{\lambda_{2} \lambda_{3}}
\end{array}\right) \\
& I^{(5)}=\frac{1}{30}\left(\begin{array}{l}
\varepsilon_{i_{1} i_{2} i_{3}} \delta_{i_{4} i_{5}} \\
\varepsilon_{i_{1} i_{2} i_{4}} \delta_{i_{3}} i_{5} \\
\varepsilon_{i_{1} i_{2} i_{5}} \delta_{i_{3} i_{4}} \\
\varepsilon_{i_{1} i_{3} i_{4}} \delta_{i_{2} i_{5}} \\
\varepsilon_{i_{1} i_{3} i_{5}} \delta_{i_{2} i_{4}} \\
\varepsilon_{i_{1} i_{4} i_{5}} \delta_{i_{2} i_{3}}
\end{array}\right)^{T}\left(\begin{array}{cccccc}
3 & -1 & -1 & 1 & 1 & 0 \\
-1 & 3 & -1 & -1 & 0 & 1 \\
-1 & -1 & 3 & 0 & -1 & -1 \\
1 & -1 & 0 & 3 & -1 & 1 \\
1 & 0 & -1 & -1 & 3 & -1 \\
0 & 1 & -1 & 1 & -1 & 3
\end{array}\right) \\
& \times\left(\begin{array}{l}
\varepsilon_{\lambda_{1} \lambda_{2} \lambda_{3}} \delta_{\lambda_{4} \lambda_{5}} \\
\varepsilon_{\lambda_{1} \lambda_{2} \lambda_{4}} \delta_{\lambda_{3} \lambda_{5}} \\
\varepsilon_{\lambda_{1} \lambda_{2} \lambda_{5}} \delta_{\lambda_{3} \lambda_{4}} \\
\varepsilon_{\lambda_{1} \lambda_{3} \lambda_{4}} \delta_{\lambda_{2} \lambda_{5}} \\
\varepsilon_{\lambda_{1} \lambda_{3} \lambda_{5}} \delta_{\lambda_{2} \lambda_{4}} \\
\varepsilon_{\lambda_{1} \lambda_{4} \lambda_{5}} \delta_{\lambda_{2} \lambda_{3}}
\end{array}\right)
\end{aligned}
$$

where $\delta$ and $\varepsilon$ are the Kronecker and Levi-Civita symbols respectively.

The $I^{(4)}$ tensor is used to rotationally average the magnetic correlation functions $($ e.g. $\langle\boldsymbol{\mu} \boldsymbol{\mu} \boldsymbol{\mu m}\rangle)$ and $I^{(5)}$ for the electric quadrupole ones (e.g. $\langle\boldsymbol{\mu} \boldsymbol{\mu} \boldsymbol{\mu} \boldsymbol{q}\rangle)$. For example, we have

$$
\left(\langle\mu \mu \mu \boldsymbol{m}\rangle_{\Omega}\right)_{i j k l}=\left(I^{(4)}\right)_{i j k l}^{i^{\prime} k^{\prime} l^{\prime}}\langle\boldsymbol{\mu} \mu \mu \boldsymbol{m}\rangle_{i^{\prime} j^{\prime} k^{\prime} l^{\prime}}
$$

where we used Einstein summation convention for the Cartesian indices $i, j, k$, and $l$, and $\langle\ldots\rangle_{\Omega}$ stands for rotational averaging.

\section{Appendix B sum-over-states expressions of the XROA signals}

Here, we provide the sum-over-states expressions of the three diagrams shown in Fig. 2. Assuming the the incoming pulses are plane waves, the electric contribution (Fig. 2(a)) is given by

$$
\begin{aligned}
S_{\mathrm{ROA}}^{\mathrm{elec}}\left(\omega_{\mathrm{X}}, \omega_{\mathrm{s}}, \theta, \boldsymbol{e}_{\mathrm{s}}\right)= & C \Re \sum_{c c^{\prime} v} c I_{c g}\left(\omega_{\mathrm{X}}\right) I_{v g}^{*}\left(\omega_{\mathrm{X}}-\omega_{\mathrm{s}}\right) I_{c^{\prime} g}^{*}\left(\omega_{\mathrm{X}}\right) \\
& \times\left\langle\mu_{g c^{\prime}}^{i} \mu_{c^{\prime} v}^{j \dagger} \mu_{v c}^{k} \mu_{c g}^{l \dagger}\right\rangle_{\Omega}\left(\bar{e}_{\mathrm{L}}^{i} e_{\mathrm{s}}^{j} \bar{e}_{\mathrm{s}}^{k} e_{\mathrm{L}}^{l}-\bar{e}_{\mathrm{R}}^{i} e_{\mathrm{s}}^{j} \bar{e}_{\mathrm{s}}^{k} e_{\mathrm{R}}^{l}\right)
\end{aligned}
$$

where

$$
\begin{gathered}
C=\frac{2}{\hbar^{4}}\left|\mathscr{E}_{X}\right|^{2} 4 \pi^{2}\left(\frac{\hbar \omega_{\mathrm{s}}}{2 \varepsilon_{0}}\right)^{2} \frac{N}{c} \\
I_{a b}(\omega)=\frac{1}{\omega-\omega_{a b}+i \Gamma_{a b}}
\end{gathered}
$$

The magnetic contribution given in Fig. 2(b) is summed over the permutations of the magnetic interaction

$$
\begin{aligned}
S_{\mathrm{ROA}}^{\mathrm{mag}}\left(\omega_{\mathrm{X}}, \omega_{\mathrm{s}}, \theta, e_{\mathrm{s}}\right)= & C \Re \sum_{c c^{\prime} v} I_{c g}\left(\omega_{\mathrm{X}}\right) I_{v g}^{*}\left(\omega_{\mathrm{X}}-\omega_{\mathrm{s}}\right) I_{c^{\prime} g}^{*}\left(\omega_{\mathrm{X}}\right) \\
& \times\left(\left\langle m_{g c^{\prime}}^{i} \mu_{c^{\prime} v}^{j \dagger} \mu_{v c}^{k} \mu_{c g}^{l \dagger}\right\rangle_{\Omega}\left(\bar{b}_{\mathrm{L}}^{i} e_{\mathrm{s}}^{j} \bar{e}_{\mathrm{s}}^{k} e_{\mathrm{L}}^{l}-\bar{b}_{\mathrm{R}}^{i} e_{\mathrm{s}}^{j} \bar{e}_{\mathrm{s}}^{k} e_{\mathrm{R}}^{l}\right)\right. \\
& +\left\langle\mu_{g c^{\prime}}^{i} m_{c^{\prime} v^{\dagger}}^{i \dagger} \mu_{v c}^{k} \mu_{c g}^{l \dagger}\right\rangle_{\Omega}\left(\bar{e}_{\mathrm{L}}^{i} b_{\mathrm{s}}^{j} \bar{e}_{\mathrm{s}}^{k} e_{\mathrm{L}}^{l}-\bar{e}_{\mathrm{R}}^{i} b_{\mathrm{s}}^{j} \bar{e}_{\mathrm{s}}^{k} e_{\mathrm{R}}^{l}\right) \\
& +\left\langle\mu_{g c^{\prime}}^{i} \mu_{c^{\prime} v}^{j \dagger} m_{v c}^{k} \mu_{c g}^{l \dagger}\right\rangle_{\Omega}\left(\bar{e}_{\mathrm{L}}^{i} e_{\mathrm{s}}^{j} \bar{b}_{\mathrm{s}}^{k} e_{\mathrm{L}}^{l}-\bar{e}_{\mathrm{R}}^{i} e_{\mathrm{s}}^{j} \bar{s}_{\mathrm{s}}^{k} e_{\mathrm{R}}^{l}\right) \\
& \left.+\left\langle\mu_{g c^{\prime}}^{i} \mu_{c^{\prime} v}^{j \dagger} \mu_{v c}^{k} m_{c g}^{l \dagger}\right\rangle_{\Omega}\left(\bar{e}_{\mathrm{L}}^{i} e_{\mathrm{s}}^{j} \bar{e}_{\mathrm{s}}^{k} b_{\mathrm{L}}^{l}-\bar{e}_{\mathrm{R}}^{i} e_{\mathrm{s}}^{j} \bar{e}_{\mathrm{s}}^{k} b_{\mathrm{R}}^{l}\right)\right)
\end{aligned}
$$

Finally, the quadrupole contribution is given in the diagram in Fig. 2(c)

$$
\begin{aligned}
S_{\mathrm{ROA}}^{\mathrm{quad}}\left(\omega_{\mathrm{X}}, \omega_{\mathrm{s}}, \theta, \boldsymbol{e}_{\mathrm{s}}\right)=C \Re \sum_{c^{\prime} v} I_{c g}\left(\omega_{\mathrm{X}}\right) I_{v g}^{*}\left(\omega_{\mathrm{X}}-\omega_{\mathrm{s}}\right) I_{c^{\prime} g}^{*}\left(\omega_{\mathrm{X}}\right) \\
\times\left(\left\langle q_{g c^{\prime}}^{i j} \mu_{c^{\prime}{ }^{\prime}{ }^{\prime \dagger}} \mu_{v c}^{l} \mu_{c g}^{m \dagger}\right\rangle_{\Omega}(-i) k^{j}\left(\bar{e}_{\mathrm{L}}^{i} e_{\mathrm{s}}^{j} e_{\mathrm{s}}^{k} e_{\mathrm{L}}^{l}-\bar{e}_{\mathrm{R}}^{i} e_{\mathrm{s}}^{j} \bar{e}_{\mathrm{s}}^{k} e_{\mathrm{R}}^{l}\right)\right) \\
+\left\langle\mu_{g c^{\prime}}^{i} q_{c^{\prime} v}^{j \dagger} \mu_{v c}^{l} \mu_{c g}^{m \dagger}\right\rangle_{\Omega} i k_{\mathrm{s}}^{k}\left(\bar{e}_{\mathrm{L}}^{i} e_{\mathrm{s}}^{j} \bar{e}_{\mathrm{s}}^{k} e_{\mathrm{L}}^{l}-\bar{e}_{\mathrm{R}}^{i} e_{\mathrm{s}}^{j} \bar{e}_{\mathrm{s}}^{k} e_{\mathrm{R}}^{l}\right) \\
+\left\langle\mu_{g c^{\prime}}^{i} \mu_{c^{\prime} v}^{j \dagger} v_{v c}^{k l} \mu_{c g}^{m \dagger}\right\rangle_{\Omega}(-i) k_{\mathrm{s}}^{l}\left(\bar{e}_{\mathrm{L}}^{i} e_{\mathrm{s}}^{j} \bar{e}_{\mathrm{s}}^{k} e_{\mathrm{L}}^{l}-\bar{e}_{\mathrm{R}}^{i} e_{\mathrm{s}}^{j} \bar{e}_{\mathrm{s}}^{k} e_{\mathrm{R}}^{l}\right) \\
+\left\langle\mu_{g c^{\prime}}^{i} \mu_{c^{\prime} v}^{j \dagger} \mu_{v c}^{k} q_{c g}^{l m \dagger}\right\rangle_{\Omega} i k^{m}\left(\bar{e}_{\mathrm{L}}^{i} e_{\mathrm{s}}^{j} \bar{e}_{\mathrm{s}}^{k} e_{\mathrm{L}}^{l}-\bar{e}_{\mathrm{R}}^{i} e_{\mathrm{s}}^{j} \bar{e}_{\mathrm{s}}^{k} e_{\mathrm{R}}^{l}\right)
\end{aligned}
$$

Note that the variable $k$ and $k_{\mathrm{s}}$ are the wavevectors of the incoming and outgoing photons respectively while the superscripts $k$ refer to the dummy summation index over cartesian components. The four-point correlation function of matter can be expanded in eigenstates. Chiral signals are measured in ensembles of randomly oriented chiral molecules. Thus, the four-point matter correlation function, eqn (17) and (18), must be rotationally averaged (see Appendix A). We use the following identities to simplify the polarization vector differences:

$$
\begin{gathered}
\bar{e}_{\mathrm{L}}^{i} e_{\mathrm{L}}^{l}-\bar{e}_{\mathrm{R}}^{i} e_{\mathrm{R}}^{l}=-i \varepsilon^{\lim \hat{k}^{m}} \\
\bar{b}_{\mathrm{L}}^{i} e_{\mathrm{L}}^{l}-\bar{b}_{\mathrm{R}}^{i} e_{\mathrm{R}}^{l}=i\left(\delta^{i l}-\hat{k}^{i} \hat{k}^{l}\right)
\end{gathered}
$$

\section{Appendix $C$ origin dependence upon coordinate change}

The transition matrix elements can be computed either in the length or in the velocity gauge, denoted as $r$ and $p$ respectively. Their transformations under a coordinate translation a from the origin $\mathbf{O}$ are given by

$$
\begin{gathered}
\boldsymbol{\mu}_{i j}^{\mathrm{r}}(\mathbf{O}+\mathbf{a})=\boldsymbol{\mu}_{i j}^{\mathrm{r}}(\mathbf{O}) \\
\boldsymbol{m}_{i j}(\mathbf{O}+\mathbf{a})=\boldsymbol{m}_{i j}^{\mathrm{r}}(\mathbf{O})+\left(\frac{1}{2} \mathbf{a} \times \mathbf{p}\right)_{i j}
\end{gathered}
$$




$$
\boldsymbol{q}_{i j}^{r}(\mathbf{O}+\mathbf{a})=\boldsymbol{q}_{i j}^{r}(\mathbf{O})-\frac{3}{2}\left(\mathbf{a} \otimes \boldsymbol{\mu}_{i j}+\boldsymbol{\mu}_{i j} \otimes \mathbf{a}\right)+\mathbf{a} \cdot \boldsymbol{\mu}_{i j} \mathbb{1}
$$

$$
\begin{gathered}
\boldsymbol{\mu}_{i j}^{\mathrm{p}}(\mathbf{O}+\mathbf{a})=\boldsymbol{\mu}_{i j}^{\mathrm{P}}(\mathbf{O}) \\
\boldsymbol{q}_{i j}^{\mathrm{P}}(\mathbf{O}+\mathbf{a})=\boldsymbol{q}_{i j}^{\mathrm{P}}(\mathbf{O})-\left(\mathbf{a} \otimes \boldsymbol{\mu}_{i j}+\boldsymbol{\mu}_{i j} \otimes \mathbf{a}\right)
\end{gathered}
$$

The magnetic dipole remains unchanged in the length and velocity gauge. ${ }^{51}$ The ROA spectroscopic signal is origin independent with truncated basis sets only in the velocity gauge. Thus the velocity gauge has been used to compute the signals. ${ }^{52}$

\section{Conflicts of interest}

There are no conflicts to declare.

\section{Acknowledgements}

The support of the Chemical Sciences, Geosciences, and Biosciences division, Office of Basic Energy Sciences, Office of Science, U.S. Deparment of Energy through Award No. DE-FG0204ER15571 and of the National Science Foundation (Grant No CHE-1663822) is gratefully acknowledged. J. R was supported by the DOE grant. Y. Z. would like to thank Prof. Jochen Autschbach for helpful discussions.

\section{References}

1 Y. Ding, Z. Huang, D. Ratner, P. Bucksbaum and H. Merdji, Generation of attosecond X-ray pulses with a multicycle twocolor enhanced self-amplified spontaneous emission scheme, Phys. Rev. Spec. Top.-Accel. Beams, 2009, 12, 060703.

2 M. Chini, K. Zhao and Z. Chang, The generation, characterization and applications of broadband isolated attosecond pulses, Nat. Photonics, 2014, 8, 178.

3 J. D. Biggs, Y. Zhang, D. Healion and S. Mukamel, Twodimensional stimulated resonance Raman spectroscopy of molecules with broadband X-ray pulses, J. Chem. Phys., 2012, 136(17), 174117.

4 Y. Zhang, J. D. Biggs and S. Mukamel, Understanding excitation energy transfer in metalloporphyrin heterodimers with different linkers, bonding structures, and geometries through stimulated X-ray Raman spectroscopy, J. Mod. Opt., 2014, 61, 558-567.

5 S.-K. Son, H. N. Chapman and R. Santra, Determination of multiwavelength anomalous diffraction coefficients at high x-ray intensity, J. Phys. B: At., Mol. Opt. Phys., 2013, 46, 164015.

6 K. Tamasaku, E. Shigemasa, Y. Inubushi, T. Katayama, K. Sawada, H. Yumoto, H. Ohashi, H. Mimura, M. Yabashi, K. Yamauchi, et al., X-ray two-photon absorption competing against single and sequential multiphoton processes, Nat. Photonics, 2014, 8, 313.
7 N. Berova, K. Nakanashi and R. W. Woody, in Circular Dichroism, Principles and Applications, Wiley, 2000.

8 A. Lázár, J. G. Ángyán, M. Hollósi, P. Huszthy and P. R. Surján, Theoretical CD spectrum calculations of the crown-ether aralkyl-ammonium salt complex, Chirality, 2002, 14, 377-385.

9 L. Ashton, P. Pudney, E. Blanch and G. Yakubov, Understanding glycoprotein behaviours using Raman and Raman optical activity spectroscopies: Characterising the entanglement induced conformational changes in oligosaccharide chains of mucin, Adv. Colloid Interface Sci., 2013, 199, 66-77.

10 L. D. Barron, The development of biomolecular Raman optical activity spectroscopy, Biomed. Spectrosc. Imaging, 2015, 4, 223-253.

11 Y. Tang and A. E. Cohen, Optical chirality and its interaction with matter, Phys. Rev. Lett., 2010, 104, 163901.

12 J. R. Rouxel, V. Y. Chernyak and S. Mukamel, Non-local realspace analysis of chiral optical signals, Chem. Sci., 2016, 7, 6824-6831.

$13 \mathrm{H}$. Zhang and A. Govorov, Giant circular dichroism of a molecule in a region of strong plasmon resonances between two neighboring gold nanocrystals, Phys. Rev. B: Condens. Matter Mater. Phys., 2013, 87, 075410.

14 M. Schäferling, D. Dregely, M. Hentschel and H. Giessen, Tailoring enhanced optical chirality: design principles for chiral plasmonic nanostructures, Phys. Rev. X, 2012, 2, 031010.

15 L. A. Nafie, Infrared and Raman vibrational optical activity: theoretical and experimental aspects, Annu. Rev. Phys. Chem., 1997, 48, 357-386.

16 L. Barron, in Molecular light scattering and optical activity, Cambridge, 2004.

17 J. Kapitán, L. D. Barron and L. Hecht, A novel Raman optical activity instrument operating in the deep ultraviolet spectral region, J. Raman Spectrosc., 2015, 46, 392-399.

18 L. Barron and A. Buckingham, Rayleigh and Raman scattering from optically active molecules, Mol. Phys., 1971, 20, 1111-1119.

19 L. Barron, M. Bogaard and A. Buckingham, Raman scattering of circularly polarized light by optically active molecules, J. Am. Chem. Soc., 1973, 95, 603-605.

20 T. Dartigalongue and F. Hache, Time-resolved circular dichroism in carbonmonoxy-myoglobin: The central role of the proximal histidine, Chirality, 2006, 18, 273-278.

21 F. Hache, M.-C. Schanne-Klein, H. Mesnil, M. Alexandre, G. Lemercier and C. Andraud, Nonlinear optical activity in chiral molecules: surface second harmonic generation and nonlinear circular dichroism, C. R. Phys., 2002, 3, 429-437.

22 A. Salam, Molecular quantum electrodynamics: long-range intermolecular interactions, John Wiley \& Sons, 2010.

23 D. Abramavicius, B. Palmieri, D. V. Voronine, F. Sanda and S. Mukamel, Coherent multidimensional optical spectroscopy of excitons in molecular aggregates; quasiparticle versus supermolecule perspectives, Chem. Rev., 2009, 109, 2350-2408. 
24 A. D. Becke, Density-functional thermochemistry. III. The role of exact exchange, J. Chem. Phys., 1993, 98, 5648-5652.

25 P. J. Stephens, F. J. Devlin, C. F. Chabalowski and M. J. Frisch, $A b$ Initio Calculation of Vibrational Absorption and Circular Dichroism Spectra Using Density Functional Force Fields, J. Phys. Chem., 1994, 98, 11623-11627.

26 T. H. Dunning, Gaussian Basis Sets for Use in Correlated Molecular Calculations. I. The Atoms Boron through Neon and Hydrogen, J. Chem. Phys., 1989, 90, 1007-1023.

27 R. A. Kendall, T. H. Dunning Jr and R. J. Harrison, Electron affinities of the firstâĂRrow atoms revisited. Systematic basis sets and wave functions, J. Chem. Phys., 1992, 96(9), 67966806.

28 M. J. Frisch, G. W. Trucks, H. B. Schlegel, G. E. Scuseria, M. A. Robb, J. R. Cheeseman, G. Scalmani, V. Barone, G. A. Petersson, H. Nakatsuji, X. Li, M. Caricato, A. Marenich, J. Bloino, B. G. Janesko, R. Gomperts, B. Mennucci, H. P. Hratchian, J. V. Ortiz, A. F. Izmaylov, J. L. Sonnenberg, D. Williams-Young, F. Ding, F. Lipparini, F. Egidi, J. Goings, B. Peng, A. Petrone, T. Henderson, D. Ranasinghe, V. G. Zakrzewski, J. Gao, N. Rega, G. Zheng, W. Liang, M. Hada, M. Ehara, K. Toyota, R. Fukuda, J. Hasegawa, M. Ishida, T. Nakajima, Y. Honda, O. Kitao, H. Nakai, T. Vreven, K. Throssell, J. A. Montgomery Jr, J. E. Peralta, F. Ogliaro, M. Bearpark, J. J. Heyd, E. Brothers, K. N. Kudin, V. N. Staroverov, T. Keith, R. Kobayashi, J. Normand, K. Raghavachari, A. Rendell, J. C. Burant, S. S. Iyengar, J. Tomasi, M. Cossi, J. M. Millam, M. Klene, C. Adamo, R. Cammi, J. W. Ochterski, R. L. Martin, K. Morokuma, O. Farkas, J. B. Foresman, and D. J. Fox, Gaussian 09, Revision C.01, Gaussian, Inc., Wallingford CT, 2009.

29 S. Miertus, E. Scrocco and J. Tomasi, Electrostatic Interaction of a Solute with a Continuum. A Direct Utilization of $a b$ initio Molecular Potentials for the Prevision of Solvent Effects, Chem. Phys., 1981, 55(1), 117129.

30 S. Miertus and J. Tomasi, Approximate Evaluations of the Electrostatic Free Energy and Internal Energy Changes in Solution Processes, Chem. Phys., 1982, 65, 239.

31 J. L. Pascual-Ahuir, E. Silla and I. Tuñón, GEPOL: An improved description of molecular-surfaces. 3. A new algorithm for the computation of a solvent-excluding surface, J. Comput. Chem., 1994, 15(10), 1127-1138.

32 J. Autschbach, T. Ziegler, S. J. A. van Gisbergen and E. J. Baerends, Chiroptical Properties from Timedependent Density Functional Theory. I. Circular Dichroism Spectra of Organic Molecules, J. Chem. Phys., 2002, 116, 6930-6940.

33 J. Autschbach and T. Ziegler, Calculating Molecular Electric and Magnetic Properties from Time-dependent Density Functional Response Theory, J. Chem. Phys., 2002, 116, 891-896.

34 J. Autschbach, Time-Dependent Density Functional Theory for Calculating Origin-Independent Optical Rotation and Rotatory Strength Tensors, ChemPhysChem, 2011, 12, 3224-3235.
35 M. Stener, G. Fronzoni and M. de Simone, Time Dependent Density Functional Theory of Core Electrons Excitations, Chem. Phys. Lett., 2003, 373, 115-123.

36 N. A. Besley and A. Noble, Time-Dependent Density Functional Theory Study of the X-ray Absorption Spectroscopy of Acetylene, Ethylene, and Benzene on Si(100), J. Phys. Chem. C, 2007, 111, 3333-3340.

37 S. DeBeer-George, T. Petrenko and F. Neese, Timedependent Density Functional Calculations of Ligand K-edge X-ray Absorption Spectra, Inorg. Chim. Acta, 2008, 361, 965-972.

38 W. Liang, S. A. Fischer, M. J. Frisch and X. Li, EnergySpecific Linear Response TDHF/TDDFT for Calculating High-Energy Excited States, J. Chem. Theory Comput., 2011, 7, 3540-3547.

39 K. Lopata, B. E. V. Kuiken, M. Khalil and N. Govind, LinearResponse and Real-Time Time-Dependent Density Functional Theory Studies of Core-Level Near-Edge XRay Absorption, J. Chem. Theory Comput., 2012, 8, 3284-3292. 40 Y. Zhang, J. D. Biggs, D. Healion, N. Govind and S. Mukamel, Core and Valence Excitations in Resonant X-ray Spectroscopy Using Restricted Excitation Window Time-dependent Density Functional Theory, J. Chem. Phys., 2012, 137, 194306.

41 M. Valiev, E. Bylaska, N. Govind, K. Kowalski, T. Straatsma, H. V. Dam, D. Wang, J. Nieplocha, E. Apra, T. Windus, et al., NWChem: A Comprehensive and Scalable Opensource Solution for Large Scale Molecular Simulations, Comput. Phys. Commun., 2010, 181, 1477-1489.

42 I. Tamm, Relativistic interaction of elementary particles, J. Phys., 1945, 9, 449.

43 S. M. Dancoff, Non-Adiabatic Meson Theory of Nuclear Forces, Phys. Rev., 1950, 78, 382-385.

44 S. Hirata and M. Head-Gordon, Time-dependent Density Functional Theory within the Tamm-Dancoff Approximation, Chem. Phys. Lett., 1999, 314, 291-299.

45 A. Klamt and G. Schüürmann, COSMO: a new approach to dielectric screening in solvents with explicit expressions for the screening energy and its gradient, J. Chem. Soc., Perkin Trans. 2, 1993, 799.

46 M. E. Casida and M. Huix-Rotllant, Progress in timedependent density-functional theory, Annu. Rev. Phys. Chem., 2012, 63, 287-323.

47 A. Chantzis, A. D. Laurent, C. Adamo and D. Jacquemin, Is the Tamm-Dancoff approximation reliable for the calculation of absorption and fluorescence band shapes?, J. Chem. Theory Comput., 2013, 9, 4517-4525.

48 S. DeBeer George and F. Neese, Calibration of scalar relativistic density functional theory for the calculation of sulfur K-edge X-ray absorption spectra, Inorg. Chem., 2010, 49, 1849-1853.

49 M. J. Peach, M. J. Williamson and D. J. Tozer, Influence of triplet instabilities in TDDFT, J. Chem. Theory Comput., 2011, 7, 3578-3585.

50 C. Hu, O. Sugino and K. Watanabe, Performance of TammDancoff approximation on nonadiabatic couplings by timedependent density functional theory, J. Chem. Phys., 2014, 140, 054106. 
51 L. K. Sørensen, R. Lindh and M. Lundberg, What do we approximate and what are the consequences in perturbation theory?, arXiv preprint arXiv:1608.023992016.

52 S. Luber and M. Reiher, Raman optical activity spectra of chiral transition metal complexes, Chem. Phys., 2008, 346, 212-223.

53 F. London, Théorie quantique des courants interatomiques dans les combinaisons aromatiques, J. Phys. Radium, 1937, 8, 397.

54 T. Helgaker, K. Ruud, K. L. Bak, P. Jørgensen and J. Olsen, Vibrational Raman optical activity calculations using London atomic orbitals, Faraday Discuss., 1994, 99, 165-180.

55 M. Krykunov and J. Autschbach, Calculation of originindependent optical rotation tensor components in approximate time-dependent density functional theory, J. Chem. Phys., 2006, 125(3), 034102.

56 Y. Zhang, J. R. Rouxel, J. Autschbach, N. Govind and S. Mukamel, X-ray circular dichroism signals: a unique probe of local molecular chirality, Chem. Sci., 2017, 8, 5969-5978.

57 J. Goulon, A. Rogalev, F. Wilhelm, N. Jaouen, C. GoulonGinet and C. Brouder, Optical activity probed with x-rays, $J$. Phys.: Condens. Matter, 2003, 15, S633.

58 L. Alagna, T. Prosperi, S. Turchini, J. Goulon, A. Rogalev, C. Goulon-Ginet, C. R. Natoli, R. D. Peacock and B. Stewart, X-ray natural circular dichroism, Phys. Rev. Lett., 1998, 80, 4799.

59 B. Stewart, R. D. Peacock, L. Alagna, T. Prosperi, S. Turchini, J. Goulon, A. Rogalev and C. Goulon-Ginet, Circular Dichroism at the Edge: Large X-ray Natural CD in the $1 \mathrm{~s} \rightarrow 3 \mathrm{~d}$ Pre-Edge Feature of 2 [Co (en) $\left.3 \mathrm{Cl}_{3}\right] \cdot \mathrm{NaCl} \cdot 6 \mathrm{H}_{2} \mathrm{O}, \mathrm{J}$. Am. Chem. Soc., 1999, 121, 1023310234.

60 D. P. Craig and T. Thirunamachandran, Molecular quantum electrodynamics: an introduction to radiation-molecule interactions, Courier Corporation, 1984. 